\title{
Survival factors of Burkitts Lymphoma patients at discharge: the case of St. Mary's Hospital Lacor in Northern Uganda
}

\author{
Mbazira Mike ${ }^{1}$, Wesonga Ronald ${ }^{1,}$, Nabugoomu Fabian ${ }^{2}$ \\ ${ }^{1}$ School of Statistics and Planning, Makerere University, Kampala, Uganda \\ ${ }^{2}$ Kyambogo University, Kampala, Uganda
}

Email address:

mbamicael@yahoo.com(M. Mike),wesonga@wesonga.com(W. Ronald), fnabugoomu@yahoo.com(N. Fabian)

\section{To cite this article:}

Mbazira Mike, Wesonga Ronald, Nabugoomu Fabian. Survival Factors of Burkitts Lymphoma Patients at Discharge: The Case of St. Mary's Hospital Lacor in Northern Uganda. American Journal of Health Research. Vol. 2, No. 1, 2014, pp. 9-14. doi: 10.11648/j.ajhr.20140201.12

\begin{abstract}
The purpose was to explore the contribution of the presenting demographic, clinical and laboratory characteristics to the survival status at discharge and duration of admission. The study used secondary data of the Burkitts Lymphoma (BL) patients who were admitted at St. Mary's Hospital Lacor between the period 2003 and 2009. Regressive methods were developed to establish the factors explaining the dependent variables. Whereas majority of the admissions were males $(60 \%)$, the proportion of females diagnosed with the cancer progressively increased more than that of males over years. Regardless of any other anatomic site involved; 74 percent of the patients had abdominal tumors, 49 percent had tumors in the face and 23 percent had tumors in the CNS. Females were more likely to have abdominal tumor involvement than males. On the other hand, males were twice more likely to have facial or CNS tumor involvement than the females. Over 80 percent of the patients were staged C or D suggesting delays in seeking for treatment. Patients with adnominal or CNS tumor involvement were more likely to be staged C or D. Seven percent died within the average admission period of 96 days. All deaths were observed among stages $C$ and $D$ patients. The duration of admission between the discharged dead and alive was significantly different. Stage C and D patients tended to have longer duration of admission. Further, older patients tended to have a longer duration of admission than the younger ones. Other variables such as sex, and site tumor involvement did not have a significant effect on the duration of admission. The duration of admission and survival status tended to influence each other. Though marginal, age at admission had a significant role to play in explaining the length of admission. The deaths before admission were observed among stage $\mathrm{C}$ and $\mathrm{D}$ patients, of which over $80 \%$ of the patients were staged. This calls in a community-level follow-up study to assess their survival. The above findings suggest an accelerated risk to death among the discharged stage $\mathrm{C}$ and $\mathrm{D}$ patients.
\end{abstract}

Keywords: Burkitts Lymphoma, Survival Status, Length of Admission, Determining Factors

\section{Introduction}

Burkitts Lymphoma(BL)is the fastest growing neoplastic tumor in man with a doubling time of about 24 hours and a growth fraction of nearly 100 percent[1]. There are several forms of BL, mainly defined according to the geographical distribution, incidence magnitude and risk factors.

The sporadic malignancy is rare worldwide while the endemic malignancy is 100 -fold more common (endemic) in equatorial Africa [2]. The endemic BL in Africa was first described in Ugandan children by Dr. Denis Burkitts in 1958 at Mulago Hospital [3]. The tumor is the most common childhood malignancy in Africa [3, 4]. The commonest sites of tumor growth are the face (jaw tumors), abdomen or both and the third most frequent site is the central nervous system $[5,6]$.

Sporadic Burkitts lymphoma (sBL) is the form subsequently described outside the African region, but morphologically similar to endemic BL and affecting mainly abdominal viscera. It can be detected at any age and no specific co-factor has been described. A third subtype of BL has been proposed based on its association with HIV infection. Though well described in the developed world and known among HIV positive adults in Africa, the childhood form of the disease among HIV positive children 
has not been well characterized. HIV associated BL can be identified in any geographical area and at all ages and is of great importance especially in sub-Saharan Africa [7].

\subsection{Survival of BL Patients}

BL treating hospitals have recorded high incidences of death among BL patients. A follow-up study of $80 \mathrm{BL}$ patients at Uganda's main Hospital (Mulago Hospital) in the early November 1966, noted that the "long-term" survival rate was approximately 1 in 5 [8]. Of these 80 patients, 36 (45\%) died in the hospital

A similar study in Nigeria noted that at the time of presenting the findings, 77.9 percent of the BL patients had defaulted (were censored), 20.7 percent had died and only 1.4 percent was known to still be alive. Only 38 percent of the recruited patients had adequate chemotherapy (4 - 6 cycles), and 23.9 percent had an initial complete remission. The mean survival of the 44 patients that died was 10.5weeks. Survival in BL cases was significantly better in patients who had adequate cycles of chemotherapy than those who had less than adequate cycles of chemotherapy [9]. Similarly, in Nigeria, majority of the patients were dying within 12 months of diagnosis[6] and [10]. This rate was markedly dependent on the extent of anatomic tumor involvement.

On contrary, in East Africa, up to 50 percent of the BL patients are reportedly cured of the disease [11, 12]) with about 25 percent enjoying 5 year disease free survival [13]. Arguably, the major factors influencing remission duration and survival in BL are disease stage at diagnosis and the quality of treatment (including completion of cycles). Other factors include patient surveillance, sanctuary effect of the Central Nervous System (CNS), disease, bone marrow involvement, pattern of relapse and resistance to chemotherapy. In a study of two comparative centers, the variation in survival was attributed to the different dosages of drugs used[14].

Response to $\mathrm{BL}$ chemotherapy is believed to be influenced by various factors. It was observed that the initial response of lymphoma cases in Mulago Hospital to chemotherapy was in part determined by the size of the tumor mass[14]. Further, the extent of tumor involvement was a critical factor in long-term prognosis[8].

The main objective of this study was to investigate the factors that explain the survival of BL patients at discharge, with the case of BL admissions at St. Mary's Hospital, Lacor in Northern Uganda. Specifically, the study sought to explore the relationship between the demographic, clinical and laboratory characteristics to the survival status at discharge and to the length of stay of BL patients in the hospitals before discharge (duration of admission).

\section{Methodology}

\subsection{Data Sources}

The study used secondary data of the $521 \mathrm{BL}$ patients who were admitted at St. Mary's Hospital Lacor between 2003 and 2009. The data were extracted from the databases of patients compiled at the hospital; specifically medical form IV and notebooks were used as sources of data. Generalized Linear Model; Regressive and Survival Analysis methods were developed to establish the factors explaining the duration of admission and survival status at discharge (dependent variables).

\subsection{Development of Standardized Variable Framework and Variable Selection Process}

The Researcher perused through the databases, the Patient Medical Forms and the Counter books and developed a comprehensive framework of the variables from the different sources; assessed their uniformity and consistency recording across patients. He selected variables thought relevant to explain the study objectives. The factors adopted in understanding the dependent variables included the region of residence at admission, age at admission, sex, and BL stage. Factors such as presenting symptoms, existence of malaria, HIV/AIDS, height, weight were not considered because they were not recorded for most of the patients. Further, socio-economic factors such as household economic status were never included because the hospital was not recorded.

\subsection{Management of Survival Times}

The study focused on two dependent variables 1) duration of admission and 2) survival status at discharge. The duration of admission was the period between when one was diagnosed and confirmed to have BL and when he/she was discharged. This period was computed in days. On the other hand, the survival status was defined as either dead or alive at discharged. The explanatory variables to the main response variables included age, sex, region of residence, BL Stage.

\subsection{Data Management and Analysis}

Generalized Linear Models were used in understanding factors paramount in explaining various attributes such as the duration of admission (number of days one remained admitted). The GLM utilized in this study included the Poisson, Negative Binomial (with and without dispersion). The base categories were defined to be females/males from Lango region with BL cancer staged $\mathrm{C}$ and are 6-9 years. The choice of the stage, region and age group base categories rose from the fact that majority of the cases were falling into the categories.

\section{Results}

\subsection{General Characteristics}

We observed an increasing number of BL patients over the study time period $(2003-2009)$ and this varied by the sex of the patients $(\mathrm{P}<=0.005)$. Figure 2 shows that during 
the period, out of every ten patients admitted, $6(60 \%)$ were males and only $4(40 \%)$ were females. A critical look at Figure 2 however shows that, whereas the cancer was predominantly common among males than females, the proportion of females diagnosed with the cancer was progressively increasing compared to that of males over years.

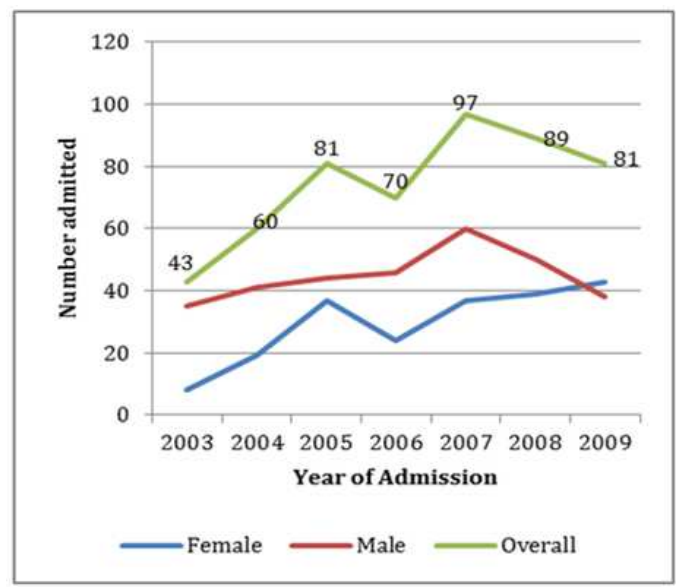

Figure 1.Enrolment over the Years by Sex

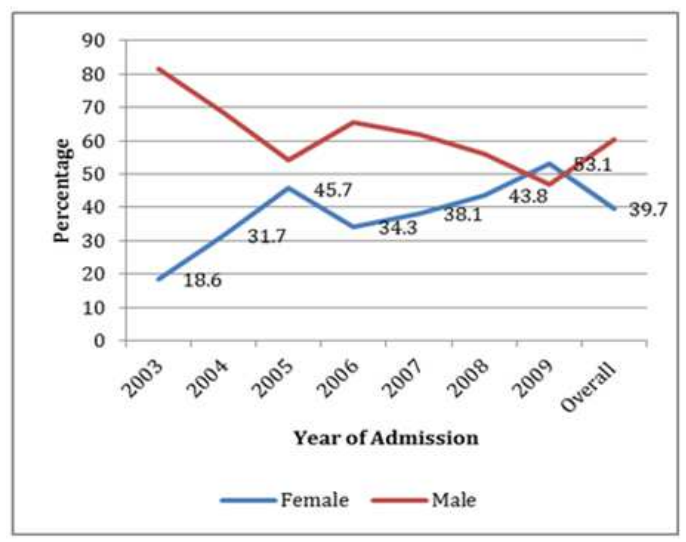

Figure 2. Comparison of Enrolments by Sex

Though the females were relatively older ( 7.5 years) than the males (7.1 years), the difference was not significant $(\mathrm{p}>0.05)$. Further, the mean age were not significantly different across the years of admission ( $p>0.05)$. Majority (49\%) of the BL patients were living/ residing in the Lango districts, 31 percent were from the districts within the Acholi region while the 20 percent were from other sub regions.

BL confirmation was mainly done using histology (57\%), cytology (34\%) and clinical (9\%). Use of these methods was not significantly related to the gender of the patients $(\mathrm{P}<=0.271)$. However, methods of diagnosis showed some significant relationship with years $(\mathrm{P}<=0.000)$ and age of the patients. Over the study years, there were observed decreasing trends in the use of cytology and clinical methods in confirming BL but with increasing trends in Histology method - Table 1. A shift in the diagnostic methods over time may be attributed to the advancements in technology, as 92 percent of the histology tissues provided a better diagnosis. Chances of having a better diagnostic tissues were 3.7 times higher with histology than cytology $(\mathrm{OR}=3.68, \mathrm{P}<=0.000)$.

Table 1. BL Diagnosis Method over the Years

\begin{tabular}{llllll}
\hline & Cytology & Histology & Clinical & Total & P-Value \\
\hline Sex & & & & & \\
Female & 30.4 & 62.8 & 6.8 & 100 & $\mathrm{P}=0.27$ \\
Male & 36.3 & 53.5 & 10.2 & 100 & \\
Year of admission & & & & \\
2003 & 67.4 & 18.6 & 14 & 100 & \\
2004 & 63.3 & 23.3 & 13.3 & 100 & \\
2005 & 72.8 & 16 & 11.1 & 100 & \\
2006 & 45.7 & 38.6 & 15.7 & 100 & $\mathrm{P}=0.00$ \\
2007 & 13.4 & 76.3 & 10.3 & 100 & \\
2008 & 4.5 & 94.4 & 1.1 & 100 & \\
2009 & 2.5 & 96.3 & 1.2 & 100 & \\
Age group & & & & & \\
2-5 & 37.8 & 51.7 & 10.5 & 100 & \\
6-9 & 36.7 & 54 & 9.3 & 100 & $\mathrm{P}=0.01$ \\
10+ & 25.5 & 70.9 & 3.6 & 100 & \\
Overall & 34 & 57.2 & 8.8 & 100 & \\
Quality of tissues taken with method & & & \\
Not & 14.8 & 3.0 & & & \\
diagnostic & & & & & \\
Poor & 9.1 & 5.1 & & & \\
diagnostic & & 91.9 & & & \\
Diagnostic & 76.1 & 100.0 & & & \\
Total & 100.0 & & & & \\
\hline
\end{tabular}

Regardless of other anatomic site involvement; 74 percent of the patients had abdominal tumor involvement, 49 percent had tumors involving the face and 23 percent had tumors in the Central Nervous System (CNS).

Males were less likely to have abdominal tumor involvement than females $(\mathrm{OR}=0.52, \mathrm{P}<=0.02)$. On the other hand, the males were twice more likely to have facial or CNS tumor involvement than the females. Those aged ten years and above were less likely to have Facial or CNS involvement as compared to those 2-5 years. Abdominal tumor involvement had no significant relationship with the age group. Whereas chances of recording abdominal and CNS tumor involvement were not significantly different across the regions of residence, the BL patients from the Acholi region were less likely to have facial tumor involvement as compared to those from Lango.

Of the BL patients whose stage was recorded, 58 percent were in stage $\mathrm{C}$ and 29 percent were stage $\mathrm{D}, 9$ percent were staged $\mathrm{B}$ and 3 percent staged A. Having over 85 percent of the patients with stages $\mathrm{C}$ or $\mathrm{D}$ suggests delayed efforts of BL patients to seek for treatments. All patients with abdominal tumor involvement or CNS were staged either C or D (advanced Stages of the cancer). Further, about 75 percent of those with facial tumor involvement were staged $\mathrm{C}$ or $\mathrm{D}$. Table 2 shows that females were at a higher risk of reporting abdominal tumor involvement that the males which explains why 92 percent of the females were grouped $\mathrm{C}$ or $\mathrm{D}$ as compared to 84 percent of the males into the same grouping $(\mathrm{C}$ or $\mathrm{D})$. 
Table 2.Relationships between BL stages, Age Groups, Anatomic Sites and Sex

\begin{tabular}{llllllll}
\hline \multicolumn{1}{c}{ Attribute/BL stage } & A & B & C & D & Total & p-value \\
\hline \multirow{4}{*}{ Age group } & $2-5$ & 3.7 & 12.6 & 52.6 & 31.1 & 100 & \\
& $6-9$ & 4.1 & 6.9 & 59.5 & 29.5 & 100 & 0.172 \\
\multirow{4}{*}{$\begin{array}{l}\text { Anatomic } \\
\text { site }\end{array}$} & $10+$ & 0 & 10 & 67.5 & 22.5 & 100 & \\
involved & Abdomen & 0 & 0 & 75.6 & 24.4 & 100 & \\
\multirow{3}{*}{ Sex } & Face & 6.1 & 18.8 & 34.5 & 40.6 & 100 & \\
& CNS & 0 & 0 & 1 & 99 & 100 & \\
& Female & 1.9 & 6.2 & 67.9 & 24.1 & 100 & \multirow{2}{*}{0.011} \\
& Male & 3.9 & 11.7 & 51.7 & 32.6 & 100 & \\
\hline & Overall & 3.1 & 9.4 & 58.4 & 29.1 & 100 & \\
\hline
\end{tabular}

\subsection{Survival at Discharge}

About seven percent of the BL admissions were discharged dead. A BL patient being discharged dead or alive was not significantly related to gender or the age group. What was clearly evident was that the deaths were only among the BL stages $\mathrm{C}$ and $\mathrm{D}$. Further, there were no observed deaths in 2003 and 2006. The chances of death among the stage $\mathrm{C}$ and $\mathrm{D}$ patients were not significantly different neither were the chances different across regions of residence nor gender of the respondent.However, those staged $\mathrm{C}$ or $\mathrm{D}$ with age bracket 2-5 years were at relatively (marginal risk of death) risk of death at discharge as compared to their counterparts who were older (above 5 years).

\subsection{Duration of Admission}

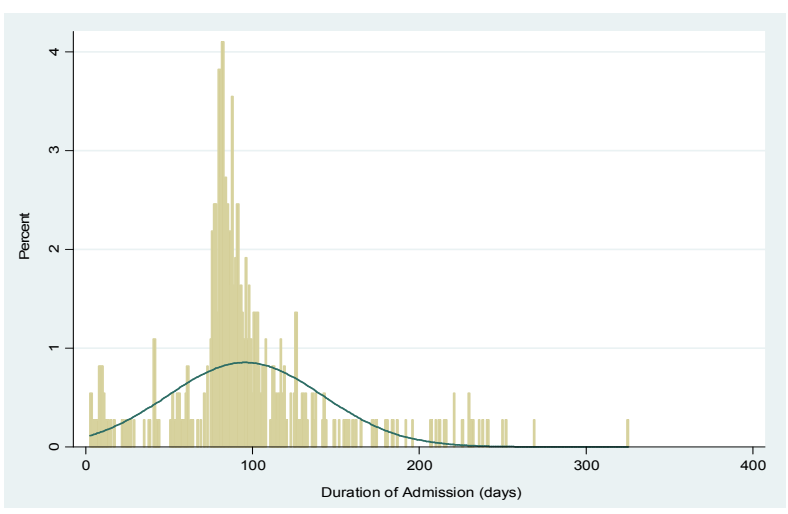

Figure 3. Average Duration of Admission

Figure 3 shows a relatively normally distributed duration of admission for the BL Patients with an average period of 96 days. The average duration of admission did not significantly differ across BL staging, Age Group, or sex of the BL patient. However, those discharged alive tended to have a significantly longer duration of admission than those who were discharged dead. On average, those discharged dead were admitted for 48 days while discharged alive were admitted for 99 days, $\mathrm{P}<0.000$. Though not significantly different, stage $\mathrm{C}$ and $\mathrm{D}$ patients tended to have longer duration of admission than the other stages (A and B).
Further, elderly patients tended to have a longer duration of admission than the younger ones.

Figure 5 shows that regardless of whether discharged dead or alive, BL patients have a $75 \%$ probability of being discharged in a period not exceeding 100 days. Patients whose BL staging was A had a 100 percent chance of being discharged in a period not exceeding 100 days.

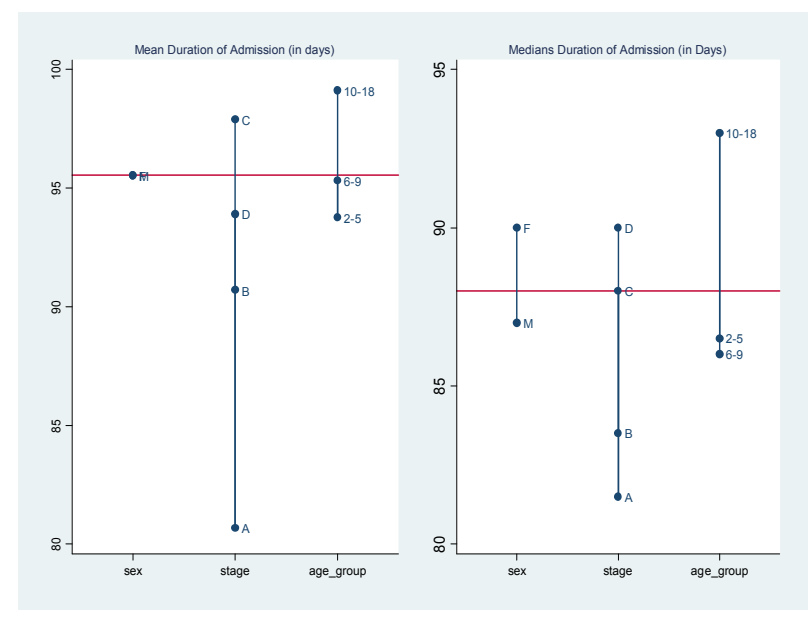

Figure 4. Average and Median Duration of Admission by BL Stage, Sex, and Survival Status
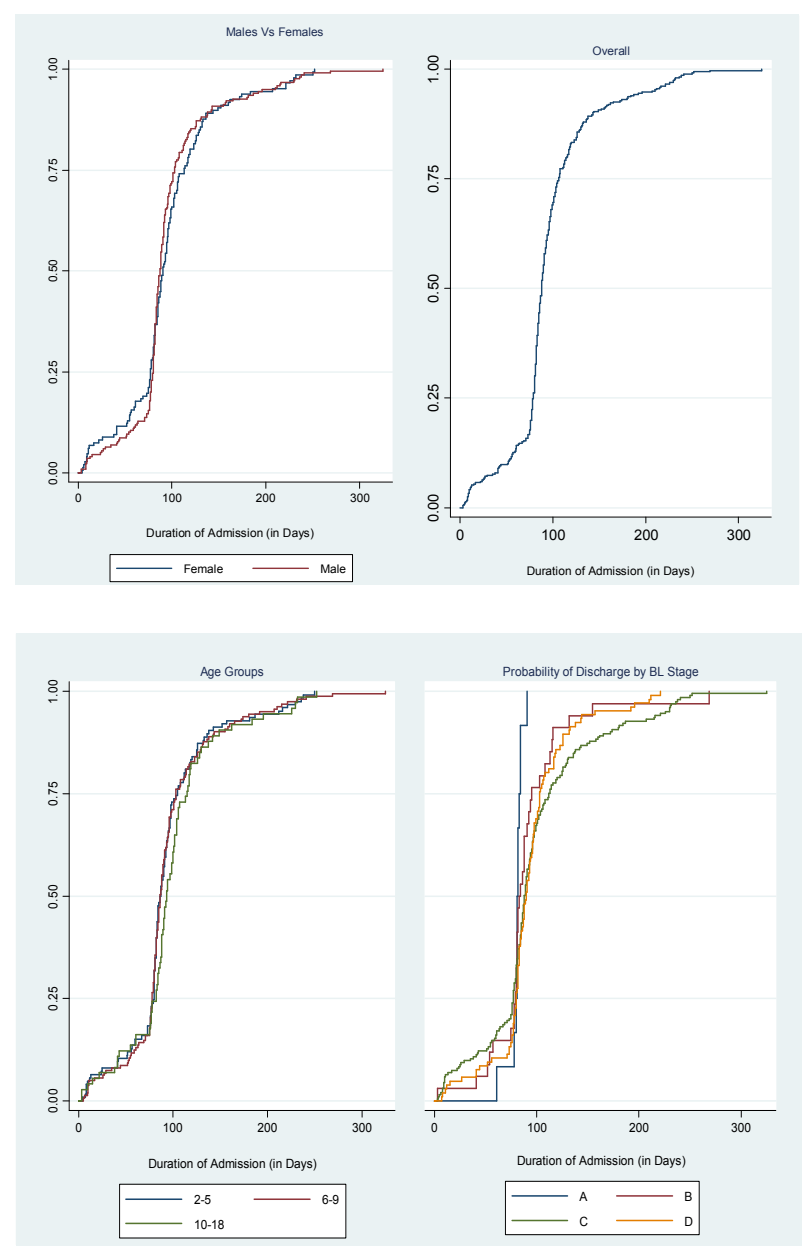

Figure 5. Kaplan Meir Survival Failure Curves for Discharge 


\section{Discussions and Conclusions}

In this paper, an exploration of significant factors in accelerating the rate at which a Burkitts Lymphoma patient admitted would be discharged from the hospital whether alive or dead was made. Secondary data on BL cases admitted at St. Mary's Hospital Lacor in Northern Uganda between 2003 and 2009 were used. Among the factors explored included one's age at admission, the degree of involvement of the cancer at the time of admission, sex, and region of residence prior to admission[8].

Of the 521 admission during the time period, 60 percent were males. However, over the years, there were increasing proportions of female admissions against the males. The patients were 7.2 years (on average) at admission. Though the average age of the females (7.6 years) seemed relatively higher than that of the males ( 7.1 years), the difference was not statistically significant at 0.05 .

Whereas the hospital was located in the central of the predominant Acholi region, majority of the patients received were from the Lango region (49\%) while the Acholi constituted 31 percent and the other areas constituted 19 percent. It's important to note that these two regions of Northern Uganda constituted about 81 percent of the BL patients received at the hospital.

Whereas in the earlier years, the hospital used Cytology to diagnose $\mathrm{BL}$, this was found to be replaced with histology over the years. Generally, there was an observed decrease in the use of cytology and clinical methods with increased use of histology. For example, 95 percent of the patients received in 2009 were diagnosed using Histology as compared to 23 percent diagnosed using the same technology in 2003. This trend could be attributed to technological advancements.

Histology was four times more likely to provide better diagnostic tissues than cytology. We also observed a consistent usage of clinical diagnosis especially among the young ones. Despite the fact that histology was a better diagnosis method than cytology, there are some situations when it was difficult or impossible to obtain a biopsy, making cytology the procedure of choice. Overall, throughout the study period; about 57 percent of the study patients were diagnosed using histology.

Most of the patients reported to the hospitals when the cancer was in its adverse stages $(58 \%$ were staged $\mathrm{C}$ while $29 \%$ were D).

Having an abdominal or CNS tumor involvement had an implication on the BL staging. All patients that had abdominal or CNS tumor involvement had BL staging $\mathrm{C}$ or $\mathrm{D}$; hence late stages of the cancer. All the patients with $\mathrm{BL}$ cancer stages A or B had facial tumors without involvement of the CNS or abdomen. Regardless of any other anatomic site involvement; 74 percent of the patients had abdominal tumors, 49 percent had tumors in the face and 23 percent had tumors in the CNS. Females were at a higher risk of reporting abdominal tumor involvement than the males which explains. About 92 percent of the females were grouped $\mathrm{C}$ or $\mathrm{D}$ as compared to 84 percent of the males into the same grouping $(\mathrm{C}$ or $\mathrm{D})$.

Within an average of 96 days of admission, 7 percent of the study patients died before they were discharged from the hospital. Those who died tended to have a significantly shorter duration of admission (dead $=48$ days, alive $=100$ days, $\mathrm{P}<=0.000$ ). No deaths at the hospital were observed among stage $\mathrm{A}$ and $\mathrm{B}$ patients. Further, there were no recorded deaths in 2003 and 2006. Though not statistically significant, stage $\mathrm{C}$ and $\mathrm{D}$ patients tended to have a relatively longer duration of admission than the other stages. Further, elderly patients tended to have a longer duration of admission than the younger ones. The patients had a $70 \%$ chance of being discharged after a period 50-100 days of admission. The chance of being discharged before duration of 50 days was less than $10 \%$. Patients whose BL staging was A had 100 percent chance of being discharged in a period not exceeding 100 days as compared to the other adverse stages.

\section{Recommendations}

Having late stage cancer admissions implies higher length of admissions for the patients which definitely constrains the hospital resources as patients are admitted for longer time periods. Findings show a clear difference in the duration of admission among stages $\mathrm{A} / \mathrm{B}$ and $\mathrm{C} / \mathrm{D}$ with the later taking longer periods. Having $75 \%$ of the total admissions requiring bed occupancy for at least 50 days brings in accommodation challenges especially in the hospital which has a total bed capacity of 482 beds. Though not common, the few cases of deaths were observed during the late stage cancers $\mathrm{C}$ and $\mathrm{D}$.

To reduce on this burden within the hospital; the following recommendations come into play

1. To undertake massive community sensitization and mobilization campaigns to improve the $\mathrm{BL}$ case spotting chances and referral to the hospitals. It has been evident that cases in the early BL stages take relatively shorter duration of admission than those in the late stages.

2. Further, observing that over 80 percent of the BL admissions were staged $\mathrm{C}$ or D (late stages), and that about seven percent of the BL patients (all in stage $\mathrm{C}$ and $\mathrm{D}$ ) had died at discharge calls for a community level follow-up study to establish the survival status of those who were discharged alive. The study can be scaled-up to incorporate all the BL patients that were discharged from the hospitals, as they are likely at the same risk to death. During this study, there were no observed cases of returnees, which may imply unrecorded high cases of death among the discharged.

3. A detailed study may be undertaken to establish why females were more likely to have abdominal tumor involvement as compared to females or why males on the other hand are likely to have facial or 
CNS tumor involvement as compared to the females.

4. Effects of other factors such as presenting symptoms, existence of malaria, HIV/AIDS, height, weight would be considered for further studies. The role played by economic factors such as economic status of the patients or families, education levels and occupation could also be explored. Further, other than the presence of BL, other studies could explore information on other competing factors such as malaria or other illnesses the patients have at the time of admission.

\section{References}

[1] O. H. Iversen, U. Iversen, J. L. Ziegler, and A. Z. Bluming, "Cell kinetics in Burkitt lymphoma," European Journal of Cancer (1965), vol. 10, pp. 155-163, 1974.

[2] I. Magrath, E. Steliarova-Foucher, S. Epelman, R. C. Ribeiro, M. Harif, C.-K. Li, R. Kebudi, S. D. Macfarlane, and S. C. Howard, "Paediatric cancer in low-income and middle-income countries," The lancet oncology, 2012.

[3] S. M. Mbulaiteye, "Burkitt Lymphoma: beyond discoveries," Infectious agents and cancer, vol. 8, pp. 1-4, 2013.

[4] R. R. Miles, S. Arnold, and M. S. Cairo, "Risk factors and treatment of childhood and adolescent Burkitt lymphoma/leukaemia," British journal of haematology, vol. 156, pp. 730-743, 2013.

[5] C. L. M. Olweny, E. Katongole Mbidde, D. Otim, S. K. Lwanga, I. T. Magrath, and J. L. Ziegler, "Long-term experience with burkitt's lymphoma in uganda," International journal of cancer, vol. 26, pp. 261-266, 1980.
[6] W. I. Aderele and A. U. Antia, "Burkitt's lymphoma in children at Ibadan-A review of 133 cases," Nig J Paediatr, vol. 6, pp. 1-14, 1979.

[7] J. Orem, E. K. Mbidde, B. Lambert, S. de Sanjose, and E. Weiderpass, "Burkitt's lymphoma in Africa, a review of the epidemiology and etiology," African health sciences, vol. 7, 2007.

[8] R. H. Morrow, M. C. Pike, and A. Kisuule, "Survival of Burkitt's lymphoma patients in Mulago Hospital, Uganda," British medical journal, vol. 4, p. 323, 1967.

[9] B. M. Kagu, O. O. Adeodu, N. O. Akinola, I. A. Adediran, and L. Salawu, "Determinants of survival in Nigerians with Burkitt's lymphoma," African journal of medicine and medical sciences, vol. 33, p. 195, 2004.

[10] C. K. O. Williams, A. O. Folami, and O. Seriki, "Patterns of treatment failure in Burkitt's lymphoma," European Journal of Cancer and Clinical Oncology, vol. 19, pp. 741-46, 1983.

[11] M. S. R. Hutt and D. Burkitt, "Geographical distribution of cancer in East Africa: a new clinicopathological approach," British medical journal, vol. 2, p. 719, 1965.

[12] S. Baik, M. Mbaziira, M. Williams, M. D. Ogwang, T. Kinyera, B. Emmanuel, J. L. Ziegler, S. J. Reynolds, and S. M. Mbulaiteye, "A case-control study of Burkitt lymphoma in East Africa: are local health facilities an appropriate source of representative controls?," Infectious agents and cancer, vol. 7, pp. 1-7, 2012.

[13] M. S. McGrath, B. Herndier, and B. Shiramizu, "Diagnosis and treatment of cancer having clonal macrophage involvement," Google Patents, 1998.

[14] D. P. Burkitt and S. K. Kyalwazi, "Spontaneous remission of African lymphoma," British journal of cancer, vol. 21, p. 14, 1967. 